\section{Time for education in cardiogenetics}

\author{
Philippe Charron, ${ }^{1}$ Perry Elliott ${ }^{2}$ \\ 'University Paris 6 (UPMC) \\ and Pitié-Salpêtrière Hospital, Paris, \\ France; \\ ${ }^{2}$ The Heart Hospital, University College, \\ London, UK
}

In all emerging fields of medicine, the discovery of new knowledge is followed by a process of translation into clinical practice. This requires the development of rapidly evolving processes for the transmission of new advances to clinicians and for implementation into everyday practice. The field of clinical cardiovascular genetics encompasses clinical diagnosis, genetic testing and counseling, and clinical management of a wide range of inherited disorders of the cardiovascular system. These include fetal cardiovascular disorders, congenital heart disease, cardiomyopathies, arrhythmias, conduction disorders and disorders of blood vessels, as well as multiple malformation syndromes, metabolic disorders, familial dyslipidemias, neuromuscular diseases, vascular disease in connective tissue disorders, haematological conditions and mitochondrial diseases. The management of such a diverse and complex group of disorders requires an integrated multi-disciplinary approach by appropriately trained and resourced healthcare professionals. In order to ensure that such services are provided for patients and families in the future, it is our view that the time has come for a much greater emphasis on clinical cardiovascular genetics in basic training and post-graduate education.

\section{The dramatic increase \\ of the knowledge}

One of the major drivers for education programs in cardiovascular genetics is the recent and major breakthrough in the discovery and understanding of the genetic basis of an increasing number of cardiovascular diseases. In some cases, notably heart muscle diseases such as hypertrophic cardiomyopathy (HCM) and arrhythmogenic right ventricular cardiomyopathy (ARVC), familial disease has been recognized for many decades, although the precise genetic defects involved continue to be discovered. In other disorders, the frequency of familial cardiovascular diseases has, until quite recently, been substantially underestimated. A typical example is dilated cardiomyopathy (DCM), a major cause of heart failure, which was previously thought to be caused by genetic mechanisms in less than $8 \%$ of cases until prospective cardiac assessment in relatives demonstrated a familial origin in $20-48 \%$ of index cases. ${ }^{1}$ Similarly, a possible familial and genetic origin of some degenerative valvular diseases has been suggested (especially in patients with bicuspid aortic valve, mitral valve prolapse but also in patients with aortic valve stenosis).

Even apparently sporadic cardiac disease may be of genetic origin. For example, molecular genetic techniques have led to the identification of mutations in patients with apparently unexplained long QT syndrome, hypertrophic cardiomyopathy and Marfan syndrome. Explanations for these sporadic phenotypes include de novo mutations in the patient (i.e. the blood cells of the parents do not carry the mutation as it was a recent mutational event) and incomplete penetrance of a mutation in one of the transmitting parents that does not express the cardiovascular disease, frequently observed in ARVC for example.

One of the greatest surprises to emerge from molecular genetic studies in cardiovascular disease is the number of genes and mutations that are regularly identified in different pathologies. For example, more than 40 genes have been reported in DCM. In some diseases, a few genes predominate (e.g. 2 in HCM, 3 in LQT, 1 in Marfan syndrome), but there are relatively few recurrent mutations resulting in huge number of mutations observed in each of these diseases ( $>700$ mutations in HCM for example).

An important consequence of the discovery that many common cardiovascular diseases have a genetic origin is the reappraisal of their natural history, particularly in relation to the age of presentation. For example, in some cases, mutation carriers may express disease only in the adult years, and sometimes quite late (in the fifth decade or beyond). This phenomenon of age related penetrance has been observed for most inherited cardiovascular diseases but especially for the cardiomyopathies. This has led to the identification of a new group of individuals frequently called preclinical carriers who carry the familial causal mutation but still do not exhibit the phenotype of the disease. A considerable research effort is underway to better characterize these relatives in order to identify potential prognostic markers (including imaging or biologic markers) and to develop early therapeutic management (including life-style modifications and early drug administration) that prevents or attenuates disease progression.

A related but different concept to age related penetrance is the variable expressivity of mutations, with a wide range of clinical findings within or between families. Although incompletely understood, the variable expressivity of many inherited cardiovascular diseases is partly related to the underlying gene/mutation (known as genotype-phenotype
Correspondence: Philippe Charron, Hôpital PitiéSalpêtrière, 47 bvd de l'Hôpital, 75013 Paris, France.

Tel. $+33.0142 .161347-+33.0142 .165535$ -

Fax: +33.0142 .161364$

E-mail: philippe.charron@psl.aphp.fr

Key words: education, clinical cardiovascular genetics, cardiovascular diseases.

Received for publication: 8 June 2011. Accepted for publication: 8 June 2011.

This work is licensed under a Creative Commons Attribution NonCommercial 3.0 License (CC BYNC 3.0).

(C) Copyright P. Charron and P. Elliott, 2011

Licensee PAGEPress, Italy

Cardiogenetics 2011; 1:e2

doi:10.4081/cardiogenetics.2011.e2

relationships, as observed in LQT or HCM for example), sometimes a second mutation (4-15\% in HCM and ARVC for example), possible modifier genes and epigenetic factors.

\section{Genetic counseling and testing \\ in clinical practice}

A major objective for the clinician is to integrate new knowledge on pathogenesis and aetiology of cardiovascular disease into clinical practice in order to improve the management of the patients and their relatives. Whatever the inherited disease, the general strategy is quite similar and three main objectives are suggested.

The first objective is to give appropriate and optimal information to the family, about various questions such as the probability of a genetic disease, the mode of inheritance, the identification of relatives at risk, the natural history of the disease (including possible delayed cardiac expression), therapeutic management, modalities for follow-up and proposals for family screening. The second objective is to organize the cardiac family evaluation. In the absence of genetic data in the family a cardiac examination should be proposed to all relatives at risk, with specific considerations about the disease considering the precise examinations, the age at which examination can start, the interval for repeated examination and the age at which the follow-up can be stopped. If genetic data are available, the cardiac screening is guided by the results of the genetic testing (predictive testing).

The third objective is to discuss genetic testing. This can be appropriate in various settings such as diagnostic testing, prognostic assessment (in a patient when there are meaningful phenotype-genotype correlations), predictive testing (in a relative without cardiac expres- 
sion but at risk of disease development in the future) and sometimes prenatal testing or preimplantation testing (to prevent the transmission of the disease to offspring).

\section{Difficulties and complexity in the management of inherited cardiovascular diseases}

A range of complementary skills are necessary to provide expert care to patients and relatives with an inherited cardiac disorder. From a clinical point of view, it is obviously necessary to understand the broad spectrum of the disease and the appropriate interpretation and management of the disease at the various stages of its natural history (preclinical, presymptomatic and symptomatic). Medical practitioners should also know how to interpret genetic results and how to apply them in genetic counseling. In addition, the clinician has to understand other important ramifications of genetic testing such as legal, social, professional and psychological issues. Specific procedures are used for genetic testing such as pre-test assessment, post-test session, psychological support, especially for complex situations such as predictive testing and prenatal testing. As the interpretation of genetic testing in inherited cardiovascular disease can be extremely challenging, clinical cardiovascular disease teams need to work very closely with diagnostic laboratories that not only provide appropriate technology and high quality operating standards but also have an understanding of the pathology of specific genetic mutations. A common problem is the identification of novel missense genetic variations, the pathogenicity of which can be difficult to determine even after exhaustive clinical and laboratory analyses. The result is that many are coded as genetic variants of uncertain significance (GVUS) or that some variants which were coded as mutations are subsequently recognized as rare polymorphisms. These findings stress the fact that genetic testing should be performed by expert and certified laboratories (that are linked to clinical research in that field), in contrast with some web-access private laboratories when no specific expertise in the interpretation of the genes concerned.

\section{The impact of next generation Sequencing}

In the very near future the use of next generation sequencing (NGS) will dramatically change the practice of genetic testing. The term is used to designate new methods of sequencing that provide massive high throughput sequencing in a short time and at low cost. ${ }^{2}$ NGS can be used for the analysis of dozens of target genes for a given disease (for example 50 to 70 genes involved in the various cardiomyopathies) or for whole exome sequencing (analyzing all the exons and intron-exon boundaries of all the 18,000 genes of the genome). This approach will undoubtedly revolutionize the diagnostic approach of genomic medicine but it also poses many new challenges, not least the analysis of massive amounts of data and the interpretation of the many thousands of genetic variants that will be identified in individual patients.

\section{How to improve education \\ in cardiogenetics?}

Sub-specialty education and training is an established activity of most areas of cardiovascular medicine. We believe strongly that similar programs of post-graduate training are required in the new subspecialty of cardiovascular genetics. This is quite a challenge as programs need to take into account the needs of a wide range of different specialists including general cardiologists, cardiologists specializing in inherited cardiovascular disease, paediatric cardiologists, heart failure specialists, electrophysiologists, clinical geneticists, obstetricians, psychologists and the diversity of pathologies involved in inherited cardiovascular diseases. Fortunately there are many existing models for the delivery of complex educational programs. These include regular systematic seminars and workshops organized by national and international specialists' societies, incorporation of cardiovascular genetic modules in core training curricula and mentoring/peer support mechanisms provided by teams of committed specialists. The European Society of Cardiology working group for myocardial and pericardial diseases is in the process of developing and promoting educational activities at an international and national level. The aim is to provide a framework for continuous professional educational programs supported by web-based learning, formal courses and ultimately formal methods of assessment including competency-based frameworks, formal written or on-line assessments and case-based discussions. In anticipation of this educational program, the working group has already produced a position statement on counseling and testing in cardiomyopathies. ${ }^{3}$ We can also anticipate that educational papers published by journals such as Cardiogenetics will greatly improve the quality of the information delivered to clinicians involved in the management of inherited cardiovascular diseases. Long life to Cardiogenetics!

\section{References}

1. Hershberger RE, Siegfried JD. Update 2011: clinical and genetic issues in familial dilated cardiomyopathy. J Am Coll Cardiol 2011;57:1641-9.

2. Metzker ML. Sequencing technologies the next generation. Nat Rev Genet 2010; 11:31-46.

3. Charron P, Arad M, Arbustini E, et al. Genetic counselling and testing in cardiomyopathies: a position statement of the European Society of Cardiology Working Group on Myocardial and Pericardial Diseases. Eur Heart J 2010;31:2715-26. 